\title{
NEW INTEGRAL REPRESENTATIONS FOR THE FOX-WRIGHT FUNCTIONS AND ITS APPLICATIONS
}

\author{
KHALED MEHREZ
}

\begin{abstract}
Our aim in this paper is to derive several new integral representations of the Fox-Wright functions. In particular, we give new Laplace and Stieltjes transform for this special functions under a special restriction on parameters. From the positivity conditions for the weight in these representations, we found sufficient conditions to be imposed on the parameters of the Fox-Wright functions that it be completely monotonic. As applications, we derive a class of function related to the Fox H-functions is positive definite and an investigation of a class of the Fox H-function is non-negative. Moreover, we extended the Luke's inequalities and we establish a new Turán type inequalities for the Fox-Wright function. Finally, by appealing to each of the Luke's inequalities, two sets of two-sided bounding inequalities for the generalized Mathieu's type series are proved.
\end{abstract}

\section{INTRODUCTION}

In this paper, we use the Fox-Wright generalized hypergeometric function ${ }_{p} \Psi_{q}[$ [.] with $p$ numerator parameters $\alpha_{1}, \ldots, \alpha_{p}$ and $q$ denominator parameters $\beta_{1}, \ldots, \beta_{q}$, which are defined by [27, p. 4, Eq. (2.4)]

$$
\begin{gathered}
\left.{ }_{p} \Psi_{q}\left[\begin{array}{c}
\left(\alpha_{1}, A_{1}\right), \ldots,\left(\alpha_{p}, A_{p}\right) \\
\left(\beta_{1}, B_{1}\right), \ldots,\left(\beta_{q}, B_{q}\right)
\end{array} \mid z\right]={ }_{p} \Psi_{q}\left[\begin{array}{c}
\left(\alpha_{p}, A_{p}\right) \\
\left(\beta_{q}, B_{q}\right)
\end{array}\right) z\right]=\sum_{k=0}^{\infty} \frac{\prod_{i=1}^{p} \Gamma\left(\alpha_{l}+k A_{l}\right)}{\prod_{j=1}^{q} \Gamma\left(\beta_{l}+k B_{l}\right)} \frac{z^{k}}{k !}, \\
\left(\alpha_{i}, \beta_{j} \in \mathbb{C}, \text { and } A_{i}, B_{j} \in \mathbb{R}^{+}(i=1, \ldots, p, j=1, \ldots, q)\right)
\end{gathered}
$$

where, as usual,

$$
\mathbb{N}=\{1,2,3, \ldots\}, \mathbb{N}_{0}=\mathbb{N} \cup\{0\},
$$

$\mathbb{R}, \mathbb{R}_{+}$and $\mathbb{C}$ stand for the sets of real, positive real and complex numbers, respectively. The defining series in (1.1) converges in the whole complex $z$-plane when

$$
\Delta=\sum_{j=1}^{q} B_{j}-\sum_{i=1}^{p} A_{i}>-1
$$

when $\Delta=0$, then the series in (1.1) converges for $|z|<\nabla$, where

$$
\nabla=\left(\prod_{i=1}^{p} A_{i}^{-A_{i}}\right)\left(\prod_{j=1}^{q} B_{j}^{B_{j}}\right) .
$$

If, in the definition (1.1), we set

$$
A_{1}=\ldots=A_{p}=1 \text { and } B_{1}=\ldots=B_{q}=1,
$$

we get the relatively more familiar generalized hypergeometric function ${ }_{p} F_{q}[$.$] given by$

$$
{ }_{p} F_{q}\left[\begin{array}{c}
\alpha_{1}, \ldots, \alpha_{p} \\
\beta_{1}, \ldots, \beta_{q}
\end{array} \mid z\right]=\frac{\prod_{j=1}^{q} \Gamma\left(\beta_{j}\right)}{\prod_{i=1}^{p} \Gamma\left(\alpha_{i}\right)} p \Psi_{q}\left[\begin{array}{c}
\left(\alpha_{1}, 1\right), \ldots,\left(\alpha_{p}, 1\right) \\
\left(\beta_{1}, 1\right), \ldots,\left(\beta_{q}, 1\right)
\end{array} \mid z\right]
$$

The Fox-Wright function appeared recently as a fundamental solutions of diffusion-like equations containing fractional derivatives in time of order less than 1 . In the physical literature, such equations are in general referred to as fractional sub-diffusion equations, since they are used as model equations for the kinetic description of anomalous diffusion processes of slow type, characterized by a sub-linear growth of the variance (the mean squared displacement) with time (see for example [21]).

File: Int.Fox-Wright.tex, printed: 2017-012-11, 2.05

2010 Mathematics Subject Classification. 33C20; 33E20; 26D07; 26A42; 44A10.

Key words and phrases. Fox-Wright function, Fox's H-function, complete monotonicity, Log-convexity, Turán type inequalities, Generalized Stieltjes function, Mathieu-type series. 
The H-function was introduced by Fox in [23] as a generalized hypergeometric function defined by an integral representation in terms of the Mellin-Barnes contour integral

$$
\begin{aligned}
H_{q, p}^{m, n}\left(\left.z\right|_{\left(A_{p}, \alpha_{p}\right)} ^{\left(B_{q}, \beta_{q}\right)}\right) & =H_{q, p}^{m, n}\left(\left.z\right|_{\left(A_{1}, \alpha_{1}\right), \ldots,\left(A_{p}, \alpha_{p}\right)} ^{\left(B_{1}, \beta_{1}\right), \ldots,\left(B_{q}, \beta_{q}\right)}\right) \\
& =\frac{1}{2 i \pi} \int_{\mathcal{L}} \frac{\prod_{j=1}^{m} \Gamma\left(A_{j} s+\alpha_{j}\right) \prod_{j=1}^{n} \Gamma\left(1-\beta_{j}-B_{j} s\right)}{\prod_{j=n+1}^{q} \Gamma\left(B_{k} s+\beta_{k}\right) \prod_{j=m+1}^{p} \Gamma\left(1-\alpha_{j}-A_{j} s\right)} z^{-s} d s
\end{aligned}
$$

Here $\mathcal{L}$ is a suitable contour in $\mathbb{C}$ and $z^{-s}=\exp (-s \log |z|+i \arg (z))$, where $\log |z|$ represents the natural logarithm of $|z|$ and $\arg (z)$ is not necessarily the principal value.

The definition of the H-function is still valid when the $A_{i}$ 's and $B_{j}$ 's are positive rational numbers. Therefore, the $\mathrm{H}$-function contains, as special cases. More importantly, it contains the Fox-Wright generalized hypergeometric function defined in (1.1), the generalized Mittag-Leffler functions, etc. For example, the function ${ }_{p} \Psi_{q}[$.$] is one of these special case of H-function. By the definition (1.1) it is easily extended$ to the complex plane as follows [18, Eq. 1.31],

$$
{ }_{p} \Psi_{q}\left[\begin{array}{c}
\left(\alpha_{p}, A_{p}\right) \\
\left(\beta_{q}, B_{q}\right)
\end{array} \mid z\right]=H_{p, q+1}^{1, q}\left(-\left.z\right|_{(0,1),\left(B_{q}, 1-\beta_{q}\right)} ^{\left(A_{p}, 1-\alpha_{p}\right)}\right) .
$$

The special case for which the $\mathrm{H}$-function reduces to the Meijer G-function is when $A_{1}=\ldots=A_{p}=$ $B_{1}=\ldots=B_{q}=A, A>0$. In this case,

$$
H_{q, p}^{m, n}\left(\left.z\right|_{\left(A_{p}, \alpha_{p}\right)} ^{\left(B_{q}, \beta_{q}\right)}\right)=\frac{1}{A} G_{p, q}^{m, n}\left(\left.z^{1 / A}\right|_{\alpha_{p}} ^{B_{q}}\right) .
$$

Additionally, when setting $A_{i}=B_{j}=1$ in (10) (or $A=1$ in (1.7)), the H- and Fox-Wright functions turn readily into the Meijer G-function.

Each of the following definitions will be used in our investigation.

A real valued function $f$, defined on an interval $I$, i called completely monotonic on $I$, if $f$ has derivatives of all orders and satisfies

$$
(-1)^{n} f^{(n)}(x) \geq 0, n \in \mathbb{N}_{0}, \text { and } x \in I .
$$

The celebrated Bernstein Characterization Theorem gives a necessary and sufficient condition that the function $f$ should be completely monotonic for $0<x<\infty$ is that

$$
f(x)=\int_{0}^{\infty} e^{-x t} d \mu(t)
$$

where $\mu(t)$ is non-decreasing and the integral converges for $0<x<\infty$.

A function $f$ is said to be absolutely monotonic on an interval $I$, if $f$ has derivatives of all orders and satisfies

$$
f^{(n)}(x) \geq 0, x \in I, n \in \mathbb{N}_{0}
$$

A positive function $f$ is said to be logarithmically completely monotonic on an interval $I$ if its logarithm $\log f$ satisfies

$$
(-1)^{n}(\log f)^{(n)}(x) \geq 0, n \in \mathbb{N}, \text { and } x \in I .
$$

In [2, Theorem 1.1] and 29, Theorem 4], it was found and verified once again that a logarithmically completely monotonic function must be completely monotonic, but not conversely.

An infinitely differentiable function $f: I \longrightarrow[0, \infty)$ is called a Bernstein function on an interval $I$, if $f^{\prime}$ is completely monotonic on $I$. The Bernstein functions on $(0, \infty)$ can be characterized by [25, Theorem $3.2]$ which states that a function $f:(0, \infty) \longrightarrow[0, \infty)$ is a Bernstein function if and only if it admits the representation

$$
f(x)=a+b x+\int_{0}^{\infty}\left(1-e^{-x t}\right) d \mu(t)
$$

where $a, b \geq 0$ and $\mu$ is a measure on $(0, \infty)$ satisfying $\int_{0}^{\infty} \min \{1, t\} d \mu(t)<\infty$. The formula (6) is called the Lévy-Khintchine representation of $f$. In [26, pp.161-162, Theorem 3] and [25, Proposition 5.25], it was proved that the reciprocal of a Bernstein function is logarithmically completely monotonic. 
In [25, Definition 2.1], it was defined that a Stieltjes transform is a function $f:(0, \infty) \longrightarrow[0, \infty)$ which can be written in the following form:

$$
f(x)=\frac{a}{x}+b+\int_{0}^{\infty} \frac{d \mu(t)}{t+x}
$$

where $a, b$ are non-negative constant and $\mu$ is a non-negative measure on $(0, \infty)$ such that the integral $\int_{0}^{\infty} \frac{d \mu(t)}{t+1}<\infty$. In [2, Theorem 2.1] it was proved that a positive Stieltjes transform must be a logarithmically completely monotonic function on $(0, \infty)$, but not conversely. We define $S$ to be the class of functions representable by (1.12). Functions representable in one of the forms

$$
f(z)=a+\int_{0}^{\infty} \frac{d \mu(t)}{(z+t)^{\alpha}}=\frac{b}{z^{\alpha}}+\int_{0}^{\infty} \frac{d \nu(t)}{(1+z t)^{\alpha}},
$$

are known as generalized Stieltjes functions of order $\alpha$. Here, $\alpha>0, \mu$ and $\nu$ are non-negative measures supported on $[0, \infty), a, b \geq 0$ are constants and we always choose the principal branch of the power function. The measures $\mu$ and $\nu$ are assumed to produce convergent integrals (1.13) for each $z \in \mathbb{C} \backslash(-\infty, 0]$. We denote by $S_{\alpha}$ to be the class of functions representable by (1.13).

The present sequel to some of the aforementioned investigations is organized as follows. In Section 2 , we derive the Laplace integral representations for the Fox H-function $H_{q, p}^{p, 0}$ and for the Fox-Wright function ${ }_{p} \Psi_{q}$. We give a numbers of consequences, some monotonicity and log-convexity properties for the Fox-Wright function are researched, and an Turán type inequality are proved. In Section 3, we find the generalized Stieltjes transform representation of the Fox-Wright function ${ }_{p+1} \Psi_{p}$. As applications, we present some class of completely monotonic functions related to the Fox-Wright function. In addition, we deduce new Turán type inequalities for this special function. In Section 4 , some further applications are proved, firstly, a class of positive definite function related to the Fox H-function are given. As consequences, we find the non-negativity for a class of function involving the Fox H-function. Next, we show that the Fox-Wright function ${ }_{p} \Psi_{q}[z]$ has no real zeros and all its zeros lie in the open right half plane $\Re(z)>0$. Moreover, two-sided exponential inequalities for the Fox-Wright function are given, in particular, we gave a generalization of Luke's inequalities. Finally, by appealing to each of these two-sided exponential inequalities, two sets of two-sided bounding inequalities for generalized Mathieu's type series are proved.

\section{LAPLACE TRANSFORM REPRESENTATION AND COMPLETELY MONOTONIC FUNCTIONS FOR THE FOX-WRIGHT FUNCTIONS}

In the first main result we will need a particular case of Fox's H-function defined by

$$
H_{q, p}^{p, 0}\left(\left.z\right|_{\left(A_{p}, \alpha_{p}\right)} ^{\left(B_{q}, \beta_{q}\right)}\right)=\frac{1}{2 i \pi} \int_{\mathcal{L}} \frac{\prod_{j=1}^{p} \Gamma\left(A_{j} s+\alpha_{j}\right)}{\prod_{k=1}^{q} \Gamma\left(B_{k} s+\beta_{k}\right)} z^{-s} d s,
$$

where $A_{j}, B_{k}>0$ and $\alpha_{j}, \beta_{k}$ are real. The contour $\mathcal{L}$ can be either the left loop $\mathcal{L}_{-}$starting at $-\infty+i \alpha$ and ending at $-\infty+i \beta$ for some $\alpha<0<\beta$ such that all poles of the integrand lie inside the loop, or the right loop $\mathcal{L}_{+}$starting $\infty+i \alpha$ at and ending $\infty+i \beta$ and leaving all poles on the left, or the vertical line $\mathcal{L}_{i c}, \Re(z)=c$, traversed upward and leaving all poles of the integrand on the left. Denote the rightmost pole of the integrand by $\gamma$ :

Let

$$
\gamma=-\min _{1 \leq j \leq p}\left(\alpha_{j} / A_{j}\right)
$$

$$
\rho=\left(\prod_{j=1}^{p} A_{j}^{A_{j}}\right)\left(\prod_{k=1}^{q} B_{k}^{-B_{k}}\right), \mu=\sum_{j=1}^{q} \beta_{j}-\sum_{k=1}^{q} \alpha_{k}+\frac{p-q}{2} .
$$

Existence conditions of Fox's H-function under each choice of the contour $\mathcal{L}$ have been thoroughly considered in the book [18. Let $z>0$ and under the conditions:

$$
\sum_{j=1}^{p} A_{j}=\sum_{k=1}^{q} B_{k}, \quad \rho \leq 1
$$

we get that the function $H_{q, p}^{p, 0}(z)$ exists by means of [18, Theorem 1.1], if we choose $\mathcal{L}=\mathcal{L}_{+}$or $\mathcal{L}=\mathcal{L}_{i c}$ under the additional restriction $\mu>1$. Only the second choice of the contour ensures the existence of the Mellin transform of $H_{q, p}^{p, 0}(z)$, see [18, Theorem 2.2]. In [7, Theorem 6], the author extend the condition 
$\mu>1$ to $\mu>0$ and proved that the function $H_{q, p}^{p, 0}(z)$ is a compact support.

In the course of our investigation, one of the main tools is the following result providing the Laplace transform of the Fox's H-function $z^{-1} H_{q, p}^{p, 0}(z)$.

Theorem 1. Suppose that $\mu>0$, and $\sum_{j=1}^{p} A_{j}=\sum_{k=1}^{q} B_{k}$. Then, the following integral representation

$$
{ }_{p} \Psi_{q}\left[\begin{array}{c}
\left(\alpha_{p}, A_{p}\right) \\
\left(\beta_{q}, B_{q}\right)
\end{array} \mid z\right]=\int_{0}^{\rho} e^{z t} H_{q, p}^{p, 0}\left(\left.t\right|_{\left(A_{p}, \alpha_{p}\right)} ^{\left(B_{q}, \beta_{q}\right)}\right) \frac{d t}{t}, \quad(z \in \mathbb{R}),
$$

hold true. Moreover, the function

$$
z \mapsto{ }_{p} \Psi_{q}\left[\begin{array}{c}
\left(\alpha_{p}, A_{p}\right) \\
\left(\beta_{q}, B_{q}\right)
\end{array} \mid-z\right]
$$

is completely monotonic on $(0, \infty)$, if and only if, the function $H_{q, p}^{p, 0}(z)$ is non-negative on $(0, \rho)$.

Proof. Upon setting $k=s, k \in \mathbb{N}_{0}$ in the Mellin transform for the Fox's H-function $H_{q, p}^{p, 0}(z)$ [7. Theorem $6]$ :

$$
\frac{\prod_{i=1}^{p} \Gamma\left(A_{i} s+\alpha_{i}\right)}{\prod_{k=1}^{q} \Gamma\left(B_{k} s+\beta_{k}\right)}=\int_{0}^{\rho} H_{q, p}^{p, 0}\left(\left.t\right|_{\left(A_{p}, \alpha_{p}\right)} ^{\left(B_{q}, \beta_{q}\right)}\right) t^{s-1} d t, \Re(s)>\gamma
$$

we get

$$
\begin{aligned}
{ }_{p} \Psi_{q}\left[\begin{array}{c}
\left(\alpha_{p}, A_{p}\right) \\
\left(\beta_{q}, B_{q}\right)
\end{array} \mid z\right] & =\sum_{k=0}^{\infty} \frac{\prod_{i=1}^{p} \Gamma\left(A_{i} k+\alpha_{i}\right) z^{k}}{k ! \prod_{j=1}^{q} \Gamma\left(B_{j} k+\beta_{j}\right)} \\
& =\sum_{k=0}^{\infty} \int_{0}^{\rho} H_{q, p}^{p, 0}\left(\left.t\right|_{\left(A_{p}, \alpha_{p}\right)} ^{\left(B_{q}, \beta_{q}\right)}\right) \frac{(z t)^{k}}{k !} \frac{d t}{t} \\
& =\int_{0}^{\rho} H_{q, p}^{p, 0}\left(\left.t\right|_{\left(A_{p}, \alpha_{p}\right)} ^{\left(B_{q}, \beta_{q}\right)}\right)\left(\sum_{k=0}^{\infty} \frac{(z t)^{k}}{k !}\right) \frac{d t}{t} \\
& =\int_{0}^{\rho} e^{z t} H_{q, p}^{p, 0}\left(\left.t\right|_{\left(A_{p}, \alpha_{p}\right)} ^{\left(B_{q}, \beta_{q}\right)}\right) \frac{d t}{t} .
\end{aligned}
$$

For the exchange of the summation and integration, we use the asymptotic relation 18, Theorem 1.2, Eq. 1.94]

$$
H_{q, p}^{m, n}(z)=\theta\left(z^{-\gamma}\right),|z| \longrightarrow 0 .
$$

Now, suppose that the function is completely monotonic on $(0, \infty)$, therefore by means of Bernstein Characterization Theorem and using the fact of the uniqueness of the measure with given Laplace transform (see [32, Theorem 6.3]), we deduce that $H_{q, p}^{p, 0}(z)$ is non-negative on $(0, \rho)$, which evidently completes the proof of Theorem 1

Corollary 1. Suppose that the hypotheses of Theorem 1 are satisfied. We define the sequence $\left(\psi_{n, m}\right)_{n, m \geq 0}$ by

$$
\psi_{n, m}=\frac{\prod_{i=1}^{p} \Gamma\left(\alpha_{i}+(n+m) A_{i}\right)}{\prod_{j=1}^{q} \Gamma\left(\beta_{j}+(n+m) B_{j}\right)}, n, m \in \mathbb{N}_{0} .
$$

If $\left(H_{1}^{n}\right): \psi_{n, 2}<\psi_{n, 1}$ and $\psi_{n, 1}^{2}<\psi_{n, 0} \psi_{n, 2}$, for all $n \in \mathbb{N}_{0}$, then the function

$$
z \mapsto{ }_{p} \Psi_{q}\left[\begin{array}{c}
\left(\alpha_{p}, A_{p}\right) \\
\left(\beta_{q}, B_{q}\right)
\end{array} \mid-z\right]
$$

is completely monotonic on $(0, \infty)$, and consequently, the function $H_{q, p}^{p, 0}(z)$ is non-negative on $(0, \rho)$.

Proof. In [11, Theorem 4], the authors proved that the function ${ }_{p} \Psi_{q}\left[\begin{array}{c}\left(\alpha_{p}, A_{p}\right) \\ \left(\beta_{q}, B_{q}\right)\end{array} \mid z\right]$ satisfying the following inequality

$$
\psi_{0,0} e^{\psi_{0,1} \psi_{0,0}^{-1}|z|} \leq{ }_{p} \Psi_{q}\left[\begin{array}{c}
\left(\alpha_{p}, A_{p}\right) \\
\left(\beta_{q}, B_{q}\right)
\end{array} \mid z\right] \leq \psi_{0,0}-\psi_{0,1}\left(1-e^{|z|}\right), z \in \mathbb{R}
$$

if $\psi_{0,1}>\psi_{0,2}$ and $\psi_{0,1}^{2}<\psi_{0,0} \psi_{0,2}$. On the other hand, by the left hand side of the above inequalities, we get for $n \geq 0$

$$
(-1)^{n} \frac{d^{n}}{d z^{n}} p \Psi_{q}\left[\begin{array}{c}
\left(\alpha_{p}, A_{p}\right) \\
\left(\beta_{p}, B_{q}\right)
\end{array} \mid-z\right]={ }_{p} \Psi_{q}\left[\begin{array}{c}
\left(\alpha_{p}+n A_{p}, A_{p}\right) \\
\left(\beta_{q}+n B_{q}, B_{q}\right)
\end{array} \mid-z\right] \geq \psi_{n, 0} e^{\psi_{n, 1} \psi_{n, 0}^{-1}|z|}>0
$$


So, the function $z \mapsto{ }_{p} \Psi_{q}\left[\begin{array}{c}\left(\alpha_{p}, A_{p}\right) \\ \left(\beta_{q}, B_{q}\right)\end{array} \mid-z\right]$ is completely monotonic on $(0, \infty)$, and consequently, the function $H_{q, p}^{p, 0}(z)$ is non-negative on $(0, \rho)$, by means of Theorem 1

Corollary 2. Suppose that the hypotheses of Corollary 1 are satisfied. In addition, assume that $\lambda, \omega>0$. Then, the function

is completely monotonic on $(0, \infty)$.

$$
z \mapsto z^{-\lambda}{ }_{p+1} \Psi_{q}\left[\begin{array}{l}
(\lambda, 1),\left(a_{p}, A_{p}\right) \\
\left(b_{q}, B_{q}\right)
\end{array} \mid-\frac{\omega}{z}\right]
$$

Proof. From the integral representation [11, Eq. 7]

$$
z_{p+1}^{-\lambda} \Psi_{q}\left[\begin{array}{l}
(\lambda, 1),\left(\alpha_{p}, A_{p}\right) \\
\left(\beta_{q}, B_{q}\right)
\end{array} \mid-\frac{\omega}{z}\right]=\int_{0}^{\infty} e^{-z t} t_{p}^{\lambda-1} \Psi_{q}\left[\begin{array}{c}
\left(\alpha_{p}, A_{p}\right) \\
\left(\beta_{q}, B_{q}\right)
\end{array} \mid-\omega t\right] d t
$$

and using the fact that the function ${ }_{p} \Psi_{q}\left[\begin{array}{c}\left(\alpha_{p}, A_{p}\right) \\ \left(\beta_{q}, B_{q}\right)\end{array} \mid-z\right] d t$, is non-negative on $(0, \infty)$, we deduce that the function $z_{p+1}^{-\lambda} \Psi_{q}\left[\begin{array}{l}(\lambda, 1),\left(\alpha_{p}, A_{p}\right) \\ \left(\beta_{q}, B_{q}\right)\end{array} \mid-\frac{\omega}{z}\right]$ is completely monotonic on $(0, \infty)$.

Remark 1. a. Combining (2.15) with (1.6), we obtain

$$
H_{p, q+1}^{1, q}\left(\left.z\right|_{(0,1),\left(B_{q}, 1-\beta_{q}\right)} ^{\left(A_{p}, 1-\alpha_{p}\right)}\right)=\int_{0}^{\rho} e^{-z t} H_{q, p}^{p, 0}\left(\left.t\right|_{\left(A_{p}, \alpha_{p}\right)} ^{\left(B_{q}, \beta_{q}\right)}\right) \frac{d t}{t} .
$$

b. In view of (2.15) and (1.7), we get

$$
{ }_{p} \Psi_{p}\left[\begin{array}{c}
\left(\alpha_{p}, A\right) \\
\left(\beta_{q}, A\right)
\end{array} \mid z\right]=A^{-1} \int_{0}^{1} e^{u z} G_{p, p}^{p, 0}\left(\left.u^{1 / A}\right|_{\alpha_{p}} ^{\beta_{p}}\right) \frac{d u}{u}, \quad A>0, z \in \mathbb{R} .
$$

Letting in the above formula, the value $A=1$, we get [8, Corollary 1, Eq. 11]

$$
{ }_{p} F_{p}\left[\begin{array}{c}
\alpha_{1}, \ldots, \alpha_{p} \\
\beta_{1}, \ldots, \beta_{p}
\end{array} \mid z\right]=\prod_{j=1}^{p} \frac{\Gamma\left(\beta_{i}\right)}{\Gamma\left(\alpha_{i}\right)} \int_{0}^{1} e^{z t} G_{p, p}^{p, 0}\left(\left.t\right|_{\alpha_{p}} ^{\beta_{p}}\right) \frac{d t}{t} .
$$

Theorem 2. Let $\alpha_{i}, \beta_{i}, i=1, \ldots, p$ be a real number such that

$$
\left(H_{2}\right): 0<\alpha_{1} \leq \ldots \leq \alpha_{p}, 0<\beta_{1} \leq \ldots \leq \beta_{p}, \sum_{j=1}^{k} \beta_{j}-\sum_{j=1}^{k} \alpha_{j} \geq 0, \text { for } k=1, \ldots, p
$$

In addition, assume that $\psi=\sum_{j=1}^{p}\left(\beta_{j}-\alpha_{j}\right)>0$. Then, the function

$$
z \mapsto{ }_{p} \Psi_{p}\left[\begin{array}{c}
\left(\alpha_{p}, A\right) \\
\left(\beta_{q}, A\right)
\end{array} \mid-z\right]
$$

is completely monotonic on $(0, \infty)$.

Proof. In [5, Lemma 2], the authors proved that the function $G_{p, p}^{p, 0}\left(\left.t\right|_{\alpha_{p}} ^{\beta_{p}}\right)$ is non-negative on $(0,1)$, and since the hypotheses of thus Theorem implies the hypotheses of Theorem 1 and so we can used the integral representation (2.20). Therefore, we deduce that all prerequisites of the Bernstein Characterization Theorem for the complete monotone functions are fulfilled, that is, the function ${ }_{p} \Psi_{p}\left[\begin{array}{c}\left(\alpha_{p}, A\right) \\ \left(\beta_{q}, A\right)\end{array} \mid-z\right]$, is completely monotonic on $(0, \infty)$. It is important to mention here that there is another proof for proved the completely monotonic for the function ${ }_{p} \Psi_{p}\left[\begin{array}{c}\left(\alpha_{p}, A\right) \\ \left(\beta_{q}, A\right)\end{array} \mid-z\right]$, without using the integral representation (2.20). For this we make use the inequalities (2.18). In our case, we have

$$
\psi_{n, m}=\prod_{j=1}^{p} \frac{\Gamma\left(\alpha_{j}+(m+n) A\right)}{\Gamma\left(\beta_{j}+(m+n) A\right)} .
$$

Under the condition $\left(H_{2}\right)$, Alzer [1, Theorem 10] proved that the function

$$
\varphi: z \mapsto \prod_{j=1}^{p} \frac{\Gamma\left(\alpha_{j}+z\right)}{\Gamma\left(\beta_{j}+z\right)}
$$


is completely monotonic on $(0, \infty)$ this yields that $\varphi(A) \geq \varphi(2 A)$ and consequently $\psi_{0,1}>\psi_{0,2}$. On the other hand, Bustoz and Ismail [9] proved that the function

$$
p(z ; a, b)=\frac{\Gamma(z) \Gamma(z+a+b)}{\Gamma(z+a) \Gamma(z+b)}, a, b \geq 0,
$$

is completely monotonic on $(0, \infty)$, then the function $z \mapsto p(z ; a, b)$ is decreasing on $(0, \infty)$. Now, we choosing $a=b=A$, we obtain $p\left(\beta_{j} ; A, A\right)<p\left(\alpha_{j} ; A, A\right)$, thus implies that $\psi_{0,1}^{2}<\psi_{0,0} \psi_{0,2}$. So, from the inequality (2.18), we deduce that the function ${ }_{p} \Psi_{p}\left[\begin{array}{c}\left(\alpha_{p}, A\right) \\ \left(\beta_{p}, A\right)\end{array} \mid-z\right]$ is non-negative on $(0, \infty)$. On the other hand, for $n \in \mathbb{N}_{0}$ we have

$$
(-1)^{n} \frac{d^{n}}{d z^{n}} \Psi_{p}\left[\begin{array}{l}
\left(\alpha_{p}, A\right) \\
\left(\beta_{p}, A\right)
\end{array} \mid-z\right]={ }_{p} \Psi_{p}\left[\begin{array}{l}
\left(\alpha_{p}+n A, A\right) \\
(\beta+n A, A)
\end{array} \mid-z\right]={ }_{p} \Psi_{p}\left[\begin{array}{l}
\left(\delta_{p}, A\right) \\
\left(\lambda_{p}, A\right)
\end{array} \mid-z\right] \geq 0
$$

where $\delta_{p}=\alpha_{p}+n A$ and $\lambda_{p}=\beta_{p}+n A$ satisfying the hypothesis $\left(H_{2}\right)$. Thus implies that the function ${ }_{p} \Psi_{p}\left[\begin{array}{l}\left(\alpha_{p}+n A, A\right) \\ (\beta+n A, A)\end{array} \mid-z\right]$ is non-negative on $(0, \infty)$, and consequently the function ${ }_{p} \Psi_{p}\left[\begin{array}{l}\left(\alpha_{p}, A\right) \\ \left(\beta_{p}, A\right)\end{array} \mid-z\right]$ is completely monotonic on $(0, \infty)$. This completes the proof of Theorem 2 .

Remark 2. We see in the second proof of the above Theorem that the condition $\psi>0$ is not necessary for proved the complete monotonicity property for the function ${ }_{p} \Psi_{p}\left[\begin{array}{c}\left(\alpha_{p}, A\right) \\ \left(\beta_{p}, A\right)\end{array} \mid-z\right]$, and consequently this property is also true under the conditions hypotheses $\left(H_{2}\right)$ only, and consequently the $\mathrm{H}$-function $H_{p, p}^{p, 0}\left[\left.t\right|_{\left(A, \alpha_{p}\right)} ^{\left(A, \beta_{p}\right)}\right]$ is non-negative under thus hypotheses.

Corollary 3. Keeping the notation and constraints of Theorem 2, Then, the function

$$
A \mapsto{ }_{p} \Psi_{p}\left[\begin{array}{c}
\left(\alpha_{p}, A\right) \\
\left(\beta_{q}, A\right)
\end{array} \mid z\right],
$$

is log-convex on $(0, \infty)$ for all $z \in \mathbb{R}$. Furthermore, then the following Turán type inequality

$$
{ }_{p} \Psi_{p}\left[\begin{array}{c}
\left(\alpha_{p}, A\right) \\
\left(\beta_{q}, A\right)
\end{array} \mid z\right] p \Psi_{p}\left[\begin{array}{c}
\left(\alpha_{p}, A+2\right) \\
\left(\beta_{q}, A+2\right)
\end{array} \mid z\right]-\left({ }_{p} \Psi_{p}\left[\begin{array}{c}
\left(\alpha_{p}, A+1\right) \\
\left(\beta_{q}, A+1\right)
\end{array} \mid z\right]\right)^{2} \geq 0
$$

holds true for all $A \in(0, \infty)$ and $z \in \mathbb{R}$.

Proof. Rewriting the integral representation (2.20) in the following form:

$$
{ }_{p} \Psi_{p}\left[\begin{array}{c}
\left(\alpha_{p}, A\right) \\
\left(\beta_{q}, A\right)
\end{array} \mid z\right]=\int_{0}^{1} e^{t^{A} z} G_{p, p}^{p, 0}\left(\left.t\right|_{\alpha_{p}} ^{\beta_{p}}\right) \frac{d t}{t}, \quad A>0, z \in \mathbb{R} .
$$

Let us recall the Hölder inequality [15, p. 54], that is

$$
\int_{a}^{b}|f(t) g(t)| d t \leq\left[\int_{a}^{b}|f(t)|^{p} d t\right]^{1 / p}\left[\int_{a}^{b}|g(t)|^{p} d t\right]^{1 / q}
$$

where $p \geq 1, \frac{1}{p}+\frac{1}{q}=1, f$ and $g$ are real functions defined on $(a, b)$ and $|f|^{p},|g|^{q}$ are integrable functions on $(a, b)$. From the Hölder's inequality and integral representation (2.23) and using the fact that the function $A \mapsto x^{A}$ is convex on $(0, \infty)$ when $x>0$. For $A_{1}, A_{2}>0$ and $t \in[0,1]$, we thus get

$$
\begin{aligned}
& { }_{p} \Psi_{p}\left[\begin{array}{c}
\left(\alpha_{p}, t A_{1}+(1-t) A_{2}\right. \\
\left(\beta_{p}, t A_{1}+(1-t) A_{2}\right)
\end{array} \mid z\right]=\int_{0}^{1} e^{z u^{t A_{1}+(1-t) A_{2}}} G_{p, p}^{p, 0}\left(\left.u\right|_{\alpha_{p}} ^{\beta_{p}}\right) \frac{d u}{u} \\
& \leq \int_{0}^{1} e^{t z u^{A_{1}}} e^{(1-t) z u^{A_{2}}} G_{p, p}^{p, 0}\left(\left.u\right|_{\alpha_{p}} ^{\beta_{p}}\right) \frac{d u}{u} \\
& =\int_{0}^{1}\left[\frac{e^{z u^{A_{1}}}}{u} G_{p, p}^{p, 0}\left(\left.u\right|_{\alpha_{p}} ^{\beta_{p}}\right)\right]^{t}\left[\frac{e^{z u^{A_{2}}}}{u} G_{p, p}^{p, 0}\left(\left.u\right|_{\alpha_{p}} ^{\beta_{p}}\right)\right]^{1-t} d u \\
& \leq\left[\int_{0}^{1} e^{z u^{A_{1}}} G_{p, p}^{p, 0}\left(\left.u\right|_{\alpha_{p}} ^{\beta_{p}}\right) \frac{d u}{u}\right]^{t}\left[\int_{0}^{1} e^{z u^{A_{2}}} G_{p, p}^{p, 0}\left(\left.u\right|_{\alpha_{p}} ^{\beta_{p}}\right) \frac{d u}{u}\right]^{1-t} \\
& =\left[{ }_{p} \Psi_{p}\left[\begin{array}{c}
\left(\alpha_{p}, A_{1}\right) \\
\left(\beta_{p}, A_{1}\right)
\end{array} \mid z\right]\right]^{t}\left[{ }_{p} \Psi_{p}\left[\begin{array}{c}
\left(\alpha_{p}, A_{2}\right) \\
\left(\beta_{p}, A_{2}\right)
\end{array} \mid z\right]\right]^{1-t},
\end{aligned}
$$

and hence the required result follows. Now, choosing $A_{1}=A, A_{2}=A+2$ and $t=\frac{1}{2}$ in the above inequality we get the Turán type inequality (2.22). 
Remark 3. Suppose the hypotheses of Theorem 2 are satisfied. Repeating the same calculations in Corollary 2 we deduce that the function

$$
z \mapsto z_{p+1}^{-\lambda} \Psi_{p}\left[\begin{array}{l}
(\lambda, 1),\left(\alpha_{p}, A\right) \\
\left(\beta_{q}, A\right)
\end{array} \mid-\frac{1}{z}\right]
$$

is completely monotonic on $(0, \infty)$, and consequently, the Hypergeometric function

$$
z \mapsto z_{p+1}^{-\lambda} F_{p}\left[\begin{array}{l}
\lambda, \alpha_{1}, \ldots, \alpha_{p} \\
\beta_{1}, \ldots, \beta_{p}
\end{array} \mid-\frac{1}{z}\right]
$$

is completely monotonic on $(0, \infty)$. (see [8, Theorem 3].)

Theorem 3. The function $z \mapsto{ }_{p} \Psi_{q}\left[\begin{array}{c}\left(\alpha_{p}, A_{p}\right) \\ \left(\beta_{q}, B_{q}\right)\end{array} \mid \frac{1}{z}\right]$ admits the following Laplace integral representation

$$
{ }_{p} \Psi_{q}\left[\begin{array}{c}
\left(\alpha_{p}, A_{p}\right) \\
\left(\beta_{q}, B_{q}\right)
\end{array} \mid \frac{1}{z}\right]=\int_{0}^{\infty} e^{-z t}\left({ }_{p} \Psi_{q+1}\left[\begin{array}{c}
\left(\alpha_{p}+1, A_{p}\right) \\
\left(\beta_{q}+1, B_{q}\right),(2,1)
\end{array} \mid t\right]+\frac{\prod_{i=1}^{p} \Gamma\left(\alpha_{i}\right)}{\prod_{j=1}^{q} \Gamma\left(\beta_{j}\right)} \delta_{0}\right) d t,
$$

where $\delta_{0}$ is the Dirac measure with mass 1 concentrated at zero. Moreover, the function

$$
z \mapsto{ }_{p} \Psi_{q}\left[\begin{array}{c}
\left(\alpha_{p}, A_{p}\right) \\
\left(\beta_{q}, B_{q}\right)
\end{array} \mid \frac{1}{z}\right]
$$

is completely monotonic on $(0, \infty)$.

Proof. Straightforward calculation would yield

$$
\begin{aligned}
\int_{0}^{\infty} & e^{-z t}\left({ }_{p} \Psi_{q+1}\left[\begin{array}{c}
\left(\alpha_{p}+1, A_{p}\right) \\
\left(\beta_{q}+1, B_{q}\right),(2,1)
\end{array} \mid t\right]+\frac{\prod_{i=1}^{p} \Gamma\left(\alpha_{i}\right)}{\prod_{j=1}^{q} \Gamma\left(\beta_{j}\right)} \delta_{0}\right) d t= \\
& =\sum_{m=0}^{\infty} \frac{\prod_{i=1}^{p} \Gamma\left(\alpha_{i}+A_{i} m+1\right)}{\prod_{j=1}^{q} \Gamma\left(\beta_{i}+B_{i} m+1\right) \Gamma(m+2) m !} \int_{0}^{\infty} t^{m} e^{-z t} d t+\frac{\prod_{i=1}^{p} \Gamma\left(\alpha_{i}\right)}{\prod_{j=1}^{q} \Gamma\left(\beta_{j}\right)} \\
& =\sum_{m=0}^{\infty} \frac{\prod_{i=1}^{p} \Gamma\left(\alpha_{i}+A_{i} m+1\right)}{\prod_{j=1}^{q} \Gamma\left(\beta_{j}+B_{j} m+1\right)(m+1) ! z^{m+1}}+\frac{\prod_{i=1}^{p} \Gamma\left(\alpha_{i}\right)}{\prod_{j=1}^{q} \Gamma\left(\beta_{j}\right)} \\
& =\sum_{m=1}^{\infty} \frac{\prod_{i=1}^{p} \Gamma\left(\alpha_{i}+A_{i} m\right)}{\prod_{j=1}^{q} \Gamma\left(\beta_{j}+B_{j} m\right) m ! z^{m}}+\frac{\prod_{i=1}^{p} \Gamma\left(\alpha_{i}\right)}{\prod_{j=1}^{q} \Gamma\left(\beta_{j}\right)} \\
& =\sum_{m=0}^{\infty} \frac{\prod_{i=1}^{p} \Gamma\left(\alpha_{i}+A_{i} m\right)}{\prod_{j=1}^{q} \Gamma\left(\beta_{j}+B_{j} m\right) m ! z^{m}} \\
& ={ }_{p} \Psi_{q}\left[\begin{array}{l}
\left(\alpha_{p}, A_{p}\right) \\
\left(\beta_{q}, B_{q}\right)
\end{array} \frac{1}{z}\right] .
\end{aligned}
$$

Therefore, the integral representation (2.26) of the function Fox-Wright function ${ }_{p} \Psi_{q}\left[\begin{array}{c}\left(\alpha_{p}, A_{p}\right) \\ \left(\beta_{q}, B_{q}\right)\end{array} \mid \frac{1}{z}\right]$ is fulfilled. Simultaneously, the function ${ }_{p} \Psi_{q+1}\left[\begin{array}{l}\left(\alpha_{p}+1, A_{p}\right) \\ \left(\beta_{q}+1, B_{q}\right),(2,1)\end{array} \mid t\right]$ being positive, all prerequisites of the Bernstein Characterization Theorem for the complete monotone functions are fulfilled, that is, the function ${ }_{p} \Psi_{q}\left[\begin{array}{c}\left(\alpha_{p}, A_{p}\right) \\ \left(\beta_{q}, B_{q}\right)\end{array} \mid \frac{1}{z}\right]$ is completely monotone on $(0, \infty)$. It is important to mention here that there is another proof for the completely monotone of the Fox-Wright function ${ }_{p} \Psi_{q}\left[\begin{array}{c}\left(\alpha_{p}, A_{p}\right) \\ \left(\beta_{q}, B_{q}\right)\end{array} \mid \frac{1}{z}\right]$. By using the fact that if the function $f(x)$ is absolutely monotonic then the function $f(1 / x)$ is completely monotonic [32, p. 151], and since the function ${ }_{p} \Psi_{q}\left[\begin{array}{c}\left(\alpha_{p}, A_{p}\right) \\ \left(\beta_{q}, B_{q}\right)\end{array} \mid z\right]$ is absolutely monotonic and consequently the function ${ }_{p} \Psi_{q}\left[\begin{array}{l}\left(\alpha_{p}, A_{p}\right) \\ \left(\beta_{q}, B_{q}\right)\end{array} \mid \frac{1}{z}\right]$ is completely monotonic on $(0, \infty)$, which evidently completes the proof of Theorem 3 .

\section{Stieltjes transform Representation for the Fox-Wright Functions and its CONSEQUENCES}

In this section, we show that the Fox-Wright function

$$
{ }_{p+1} \Psi_{q}\left[\begin{array}{l}
(\sigma, 1),\left(\alpha_{p}, A_{p}\right) \\
\left(\beta_{p}, B_{p}\right)
\end{array} \mid-z\right]
$$


is a generalized Stieltjes functions of order $\sigma$. As applications, some class of logarithmically completely monotonic functions related to the Fox-Wright function are derived. Moreover, we deduce new Turán type inequalities for thus special function.

Theorem 4. Let $\sigma>0$ and $z \in \mathbb{C}$ such that $|\arg z|<\pi$ and $|z|<1$. Assume that the hypotheses of Corollary 1 are satisfied. Then, the following Stieltjes transform hold true:

$$
{ }_{p+1} \Psi_{q}\left[\begin{array}{l}
(\sigma, 1),\left(\alpha_{p}, A_{p}\right) \\
\left(\beta_{q}, B_{q}\right)
\end{array} \mid-z\right]=\int_{0}^{\rho} \frac{d \mu(t)}{(1+t z)^{\sigma}},
$$

where

$$
d \mu(t)=H_{q, p}^{p, 0}\left(\left.t\right|_{\left(A_{p}, \alpha_{p}\right)} ^{\left(B_{q}, \beta_{q}\right)}\right) \frac{d t}{t}
$$

Furthermore, the function

$$
z \mapsto{ }_{p+1} \Psi_{q}\left[\begin{array}{l}
(\sigma, 1),\left(\alpha_{p}, A_{p}\right) \\
\left(\beta_{q}, B_{q}\right)
\end{array} \mid-z\right]
$$

is completely monotonic on $(0,1)$.

Proof. Consider the right-hand side of (3.27) with $d \mu(t)$ is given by (3.28). We make use of the formula (2.16) and applying the binomial expansion to

$$
(1+z)^{-\sigma}=\sum_{k=0}^{\infty}(\sigma)_{k} \frac{(-1)^{k} z^{k}}{k !}, z \in \mathbb{C} \text { such that }|z|<1,
$$

and integrating term by term we obtain the left-hand side of (3.27). Finally, its easy to see that the function $z \mapsto{ }_{p+1} \Psi_{q}\left[\begin{array}{l}(\sigma, 1),\left(\alpha_{p}, A_{p}\right) \\ \left(\beta_{q}, B_{q}\right)\end{array} \mid-z\right]$ is completely monotonic on $(0,1)$.

Corollary 4. Let $0<\sigma \leq 1$. Assume that the hypotheses of Corollary 1 are satisfied. Then the following assertions are true:

a. The function

$$
z \mapsto{ }_{p+1} \Psi_{q}\left[\begin{array}{l}
(\sigma, 1),\left(\alpha_{p}, A_{p}\right) \\
\left(\beta_{q}, B_{q}\right)
\end{array} \mid-z\right]
$$

is logarithmically completely monotonic on $(0,1)$.

b. The function

$$
z \mapsto 1 /{ }_{p+1} \Psi_{q}\left[\begin{array}{l}
(\sigma, 1),\left(\alpha_{p}, A_{p}\right) \\
\left(\beta_{q}, B_{q}\right)
\end{array} \mid-z\right]
$$

is a Bernstein function on $(0,1)$. In particular, the function

$$
z \mapsto{ }_{p+1} \Psi_{q}\left[\begin{array}{l}
(\sigma+1,1),\left(\alpha_{p}+A_{p}, A_{p}\right) \\
\left(\beta_{q}+B_{q}, B_{q}\right)
\end{array} \mid-z\right] / p+1 \Psi_{q}\left[\begin{array}{l}
(\sigma, 1),\left(\alpha_{p}, A_{p}\right) \\
\left(\beta_{q}, B_{q}\right)
\end{array} \mid-z\right]
$$

is completely monotonic on $(0,1)$.

Proof. a. By using the fact that $S_{\alpha} \subseteq S_{\beta}$ whenever $\alpha \leq \beta$ (see [20]), we deduce

$$
{ }_{p+1} \Psi_{q}\left[\begin{array}{l}
(\sigma, 1),\left(\alpha_{p}, A_{p}\right) \\
\left(\beta_{q}, B_{q}\right)
\end{array} \mid-z\right] \in S_{1}=S,
$$

where $0<\sigma \leq 1$. On the other hand, it was proved in [2, Theorem 1.2] that the set of Stieltjes transforms $\mathcal{S} \backslash\{0\}$ is a proper subset of the class of logarithmically completely monotonic functions.

b. The result follows from Theorem 4 and Proposition 1.3 [3].

Theorem 5. Under the assumptions stated in Corollary 1. The function

$$
\sigma \mapsto \Xi(\sigma)={ }_{p+1} \Psi_{q}\left[\begin{array}{l}
(\sigma, 1),\left(\alpha_{p}, A_{p}\right) \\
\left(\beta_{q}, B_{q}\right)
\end{array} \mid z\right]
$$

is log-convex on $(0, \infty)$ for each $z \in(0,1)$. Furthermore, the following Turán type inequality

$$
{ }_{p+1} \Psi_{q}\left[\begin{array}{l}
(\sigma, 1),\left(\alpha_{p}, A_{p}\right) \\
\left(\beta_{q}, B_{q}\right)
\end{array} \mid z\right] p+1 \Psi_{q}\left[\begin{array}{l}
(\sigma+2,1),\left(\alpha_{p}, A_{p}\right) \\
\left(\beta_{q}, B_{q}\right)
\end{array} \mid z\right]-\left({ }_{p+1} \Psi_{q}\left[\begin{array}{l}
(\sigma+1,1),\left(\alpha_{p}, A_{p}\right) \\
\left(\beta_{q}, B_{q}\right)
\end{array} \mid z\right]\right)^{2} \geq 0
$$

holds true for all $\sigma \in(0, \infty)$ and $z \in(0,1)$. 
Proof. Recall the Chebyshev integral inequality [6, p. 40]: if $f, g:[a, b] \longrightarrow \mathbb{R}$ are synchoronous (both increasing or decreasing) integrable functions, and $p:[a, b] \longrightarrow \mathbb{R}$ is a positive integrable function, then

$$
\int_{a}^{b} p(t) f(t) d t \int_{a}^{b} p(t) g(t) d t \leq \int_{a}^{b} p(t) d t \int_{a}^{b} p(t) f(t) g(t) d t
$$

Note that if $f$ and $g$ are asynchronous (one is decreasing and the other is increasing), then (3.31) is reversed. Let $\sigma_{2}>\sigma_{1} \geq 0$ and arbitrary $\epsilon>0$ and we consider the functions $p, f, g:[0, \rho] \longrightarrow \mathbb{R}$ defined by:

$$
p(t)=\frac{t^{-1} H_{q, p}^{p, 0}\left(\left.t\right|_{\left(A_{p}, \alpha_{p}\right)} ^{\left(B_{q}, \beta_{q}\right)}\right)}{(1-z t)^{\sigma_{1}}}, \quad f(t)=\frac{1}{(1-z t)^{\sigma_{2}-\sigma_{1}}}, \quad g(t)=\frac{1}{(1-z t)^{\epsilon}} .
$$

Since the function $p$ is non-negative on $(0, \rho)$ and the functions $f$ and $g$ are increasing on $(0, \rho)$ if $z \in(0,1)$, we gave

$$
\Xi\left(\sigma_{1}+\epsilon\right) \Xi\left(\sigma_{2}\right) \leq \Xi\left(\sigma_{1}\right) \Xi\left(\sigma_{2}+\epsilon\right) .
$$

The above inequality is equivalent to log-convexity for the function $\sigma \mapsto \Xi(\sigma)$ on $(0, \infty)$ for each $z \in(0,1)$ (see [17, Chapter I.4]). Now, focus on the Turán type inequality (3.30). Since the function $\sigma \mapsto \Xi(\sigma)$ is $\log$-convex on $(0, \infty)$ for each $x \in(0,1)$. it follows that for all $\sigma_{1}, \sigma_{2}>0, t \in[0,1]$ and $x \in(0,1)$, we have

$$
\Xi\left(t \sigma_{1}+(1-t) \sigma_{2}\right) \leq\left[\Xi\left(\sigma_{1}\right)\right]^{t}\left[\Xi\left(\sigma_{2}\right)\right]^{1-t} .
$$

Upon setting

$$
\sigma_{1}=\sigma, \sigma_{2}=\sigma+2 \text { and } t=\frac{1}{2},
$$

the above inequality reduces to the Turán type inequality (3.30), which evidently completes the proof of Theorem 5

Remark 4. If we consider the assumptions $\left(H_{2}\right)$ in Theorem 2 we get again the results proved in Section 3 , where $q=p$ and $A=A_{p}=B_{p}$.

\section{Further Applications}

4.1. A class of positive definite functions related to the Fox $\mathbf{H}$-function. The purpose of this section is to prove a class of positive definite functions related to the Fox H-function. As an application, we derive a class of function involving the Fox H-function is non-negative. Let us remind the reader that a continuous function $f: \mathbb{R}^{d} \longrightarrow \mathbb{C}$ is called positive definite function, if for all $N \in \mathbb{N}$, all sets of pairwise distinct centers $X=\left\{x_{1}, \ldots, x_{N}\right\} \subseteq \mathbb{R}^{d}$ and $z=\left\{\xi_{1}, \ldots, \xi_{N}\right\} \subset \mathbb{C}^{N}$, the quadratic form

$$
\sum_{j=1}^{N} \sum_{k=1}^{N} \xi_{j} \bar{\xi}_{k} f\left(x_{j}-x_{k}\right)
$$

is non-negative.

Theorem 6. Let the parameters $\rho, \nu \in \mathbb{C}$, satisfy the conditions

$$
\begin{gathered}
\Re(\rho)+\Re(\nu)+\min _{1 \leq j \leq p}\left[\frac{\alpha_{j}}{A}\right]>-1, \\
\Re(\nu)>-\frac{1}{2} \text { and } \Re(\rho)+\Re(\nu)<\frac{3}{2} .
\end{gathered}
$$

Moreover, assume that the hypotheses $\left(\mathrm{H}_{2}\right)$ of Theorem 圆 are satisfies. Then the function

$$
\chi: z \mapsto z^{-(\rho+\nu)} H_{p, p+2}^{1, p}\left[\left.2 z\right|_{\left(\frac{1}{2}, \frac{\rho+\nu}{2}\right),\left(A, 1-\beta_{p}\right),\left(\frac{1}{2}, \frac{\rho-\nu}{2}\right)}\right]
$$

is positive definite function on $\mathbb{R}$.

Proof. We can write the following formula [18, Eq. (2.45), pp. 57]

$$
\int_{0}^{\infty} x^{\rho-1} J_{\nu}(z x) H_{q, p}^{m, n}\left[\left.x\right|_{\left(A_{p}, \alpha_{p}\right)} ^{\left(B_{q}, \beta_{q}\right)}\right] d x=\frac{2^{\rho-1}}{z^{\rho}} H_{q+2, p}^{m, n+1}\left[\left.\frac{2}{z}\right|_{\left(A_{p}, \alpha_{p}\right)} ^{\left(\frac{1}{2}, 1-\frac{\rho+\nu}{2}\right),\left(B_{q}, \beta_{q}\right),\left(\frac{1}{2}, 1-\frac{\rho-\nu}{2}\right)}\right]
$$

in the following form

$$
\int_{0}^{\infty} x^{\rho+\nu-1} \mathcal{J}_{\nu}(z x) H_{q, p}^{m, n}\left[\left.x\right|_{\left(A_{p}, \alpha_{p}\right)} ^{\left(B_{q}, \beta_{q}\right)}\right] d x=\frac{\Gamma(\nu+1) 2^{\rho+\nu-1}}{z^{\rho+\nu}} H_{q+2, p}^{m, n+1}\left[\left.\frac{2}{z}\right|_{\left(A_{p}, \alpha_{p}\right)} ^{\left(\frac{1}{2}, 1-\frac{\rho+\nu}{2}\right),\left(B_{q}, \beta_{q}\right),\left(\frac{1}{2}, 1-\frac{\rho-\nu}{2}\right)}\right]
$$


where

$$
\mathcal{J}_{\nu}(x)=2^{\nu} \Gamma(\nu+1) \frac{J_{\nu}(x)}{x^{\nu}}, \Re(\nu)>-\frac{1}{2}
$$

with $J_{\nu}(x)$ is the Bessel function of index $\nu$. On the other hand, as the function $\mathcal{J}_{\nu}(x)$ is positive definite function [10, Proposition 2] and the function $H_{p, p}^{p, 0}\left[\left.t\right|_{\left(A, \alpha_{p}\right)} ^{\left(A, \beta_{p}\right)}\right]$ is non-negative (Remark 2), we deduce that for any finite list of complex numbers $\xi_{1}, \ldots, \xi_{N}$ and $z_{1}, \ldots, z_{N} \in \mathbb{R}$,

$$
\begin{aligned}
& \sum_{j=1}^{N} \sum_{k=1}^{N} \xi_{j} \overline{\xi_{k}}\left(z_{j}-z_{k}\right)^{-(\rho+\nu)} H_{p+2, p}^{p, 1}\left[\left.2\left(z_{j}-z_{k}\right)^{-1}\right|_{\left(A, \alpha_{p}\right)} ^{\left(\frac{1}{2}, 1-\frac{\rho+\nu}{2}\right),\left(A, \beta_{q}\right),\left(\frac{1}{2}, 1-\frac{\rho-\nu}{2}\right)}\right]= \\
= & \frac{1}{\Gamma(\nu+1) 2^{\rho+\nu-1}} \int_{0}^{\infty} x^{\rho+\nu-1}\left[\sum_{j=1}^{N} \sum_{k=1}^{N} \xi_{j} \overline{\xi_{k}} \mathcal{J}_{\nu}\left(x z_{j}-x z_{k}\right)\right] H_{p, p}^{p, 0}\left[\left.x\right|_{\left(A, \alpha_{p}\right)} ^{\left(A, \beta_{p}\right)}\right] d x \geq 0 .
\end{aligned}
$$

Thus, implies that the function

$$
\chi_{1}: z \mapsto z^{-(\rho+\nu)} H_{p+2, p}^{p, 1}\left[\left.\frac{2}{z}\right|_{\left(A, \alpha_{p}\right)} ^{\left(\frac{1}{2}, 1-\frac{\rho+\nu}{2}\right),\left(A, \beta_{p}\right),\left(\frac{1}{2}, 1-\frac{\rho-\nu}{2}\right)}\right]
$$

is positive definite function on $\mathbb{R}$. So, the [18, Property 1.3, p. 11] completes the proof of Theorem 6 ,

Theorem 7. Let the parameters $\rho, \nu \in \mathbb{C}$, satisfy the conditions

$$
\begin{gathered}
\Re(\rho)+\Re(\nu)+\min _{1 \leq j \leq p}\left[\frac{\alpha_{j}}{A}\right]>0, \Re(\nu)>-\frac{1}{2}, \\
\Re(1-(\rho+\nu))+\max _{1 \leq j \leq p}\left(\frac{\alpha_{j}}{A}\right)<1 \text {, and } \Re(\rho)+\Re(\nu)<\frac{3}{2} .
\end{gathered}
$$

Then, the function

$$
\mathcal{K}_{p, q}^{\nu, \rho}(z)=z^{\rho+\nu-1} H_{p+2, p+2}^{p+1,1}\left[\left.8 z\right|_{\left(\frac{1}{2}, \frac{1-(\rho+\nu)}{2}\right),\left(A, \alpha_{p}\right),\left(\frac{1}{2}, 1-\frac{\rho+\nu}{2}\right)} ^{\left(\frac{1}{2}, 1-\frac{\rho+\nu}{2}\right),\left(A, \beta_{p}\right),\left(\frac{1}{2}, 1-\frac{\rho-\nu}{2}\right)}\right]
$$

is non-negative on $\mathbb{R}$.

Proof. Firstly, we proved that the function $z \longmapsto \chi(z)$ is in $L^{1}(0, \infty)$. By using the asymptotic expansion [18, Eq. 1.94, pp. 19]

$$
H_{p, q}^{m, n}\left[\left.z\right|_{\left(B_{q}, b_{q}\right)} ^{\left(A_{p}, a_{p}\right)}\right]=\theta\left(z^{c}\right),|z| \longrightarrow 0, \text { where } c=\min _{1 \leq j \leq m}\left[\frac{\Re\left(b_{j}\right)}{B_{j}}\right] .
$$

In our case $m=1, b_{1}=\frac{\rho+\nu}{2}$ and $B_{1}=\frac{1}{2}$, and consequently $c=\rho+\nu$. Thus implies that

$$
\chi(z)=\theta(1) \text { as } z \longrightarrow 0 .
$$

On the other hand, by using the asymptotic [18, Eq. 1.94, pp. 19]

$$
H_{p, q}^{m, n}\left[z \mid \begin{array}{c}
\left(A_{p}, a_{p}\right) \\
\left(B_{q}, b_{q}\right)
\end{array}\right]=\theta\left(z^{d}\right),|z| \longrightarrow \infty, \quad \text { where } d=\min _{1 \leq j \leq n}\left[\frac{\Re\left(a_{j}\right)-1}{A_{j}}\right] .
$$

In our case $n=p$ and $a_{j}=1-\alpha_{j}$ and consequently $d=-\left(\Re(\rho)+\Re(\nu)+\min _{1 \leq j \leq p} \frac{\Re\left(\alpha_{j}\right)}{A}\right)$, thus we get

$$
\chi(z)=\theta\left(z^{-(\rho+\nu+M)}\right) \text {, where } M=\min _{1 \leq j \leq p} \frac{\alpha_{j}}{A} .
$$

Now, combining (4.40) with the hypotheses (4.36) and (4.39), we deduce that the function $z \longmapsto \chi(z)$ is in $L^{1}(0, \infty)$. In addition, as $z \mapsto \mathcal{J}_{\nu}(z x)$ is an even function and using the integral representation (4.34), we deduce that $z \longmapsto \chi(z)$ is an even function, and consequently thus function is in $L^{1}(\mathbb{R})$.

Secondly, we calculate the Fourier transform of the function $z \longmapsto \chi(z)$. Since $z \longmapsto \chi(z)$ is an even function then the the Fourier transform can be written as a Hankel transform (see [22, Lemma 1.1], when $\alpha=-1 / 2)$, more precisely,

$$
\mathcal{F}(\chi)(z)=\sqrt{\frac{2}{\pi}} \int_{0}^{\infty} \chi(x) \cos (x z) d z
$$

We now make use of the following formula [18, Eq. 2.50, p. 58]

$$
\int_{0}^{\infty} x^{\rho-1} \cos (x z) H_{p, q}^{m, n}\left[\left.x\right|_{\left(B_{q}, b_{q}\right)} ^{\left(A_{p}, a_{p}\right)}\right] d x=\frac{2^{\rho-1} \sqrt{\pi}}{z^{\rho}} H_{p+2, q}^{m, n+1}\left[\left.\frac{2}{z}\right|_{\left(B_{q}, b_{q}\right)} ^{\left(\frac{1}{2}, \frac{2-\rho}{2}\right),\left(A_{p}, a_{p}\right),\left(\frac{1}{2}, \frac{1-\rho}{2}\right)}\right]
$$


where $z>0, \rho \in \mathbb{C}$ such that

$$
\Re(\rho)+\min _{1 \leq j \leq m} \Re\left(\frac{b_{j}}{B_{j}}\right)>0 \text { and } \Re(\rho)+\max _{1 \leq j \leq n} \Re\left(\frac{a_{j}-1}{A_{j}}\right)<1 .
$$

In our case $\rho \longrightarrow 1-(\rho+\nu), m=1 n=p, b_{1}=\frac{\rho+\nu}{2}$ and $B_{1}=\frac{1}{2}$, thus

$$
\Re(\rho)+\min _{1 \leq j \leq m}\left(\frac{b_{j}}{B_{j}}\right)=1>0 .
$$

Therefore,

$$
\begin{aligned}
\mathcal{F}(\chi)(z) & =2^{1-\rho-\nu} z^{\rho+\nu-1} H_{p+2, p+2}^{1, p+1}\left[\left.\frac{8}{z}\right|_{\left(\frac{1}{2}, \frac{\rho+\nu}{2}\right),\left(A, 1-\beta_{p}\right),\left(\frac{1}{2}, \frac{\rho-\nu}{2}\right)} ^{\left(\frac{1}{2}, \frac{1+\rho+\nu}{2}\right),\left(A, 1-\alpha_{p}\right),\left(\frac{1}{2}, \frac{\rho+\nu}{2}\right)}\right] \\
& =2^{1-\rho-\nu} z^{\rho+\nu-1} H_{p+2, p+2}^{p+1,1}\left[\left.8 z\right|_{\left(\frac{1}{2}, \frac{1-(\rho+\nu)}{2}\right),\left(A, \alpha_{p}\right),\left(\frac{1}{2}, 1-\frac{\rho+\nu}{2}\right)} ^{\left(\frac{1}{2}, 1-\frac{\rho+\nu}{2}\right),\left(A, \beta_{p}\right),\left(\frac{1}{2}, 1-\frac{\rho-\nu}{2}\right)}\right] .
\end{aligned}
$$

Finally, using the fact that the Fourier transform for a function in $L^{1}$ and positive definite function is non-negative ( see for example [30, theorem 6.6] or [31, Theorem 6.11, p. 74]). So, the proof is completes.

\subsection{Zeros of the Fox-Wright functions.}

Theorem 8. Keeping the notation and constraints of Corollary 1. Then, all the roots of the Fox-Wright function ${ }_{p} \Psi_{q}\left[\begin{array}{c}\left(\beta_{p}, B_{q}\right) \\ \left(\alpha_{p}, A_{p}\right)\end{array} \mid z\right]$ are in the left-hand half-plane $\Re z \leq 0$.

Proof. By using the following identity [7, Theorem 8]

$$
H_{q, p}^{p, 0}\left(\left.z\right|_{\left(\alpha_{p}, A_{p}\right)} ^{\left(\beta_{q}, B_{q}\right)}\right)=\frac{1}{\log (\rho / z)} \int_{z / \rho}^{1} H_{q, p}^{p, 0}\left(\left.\frac{z}{u}\right|_{\left(\alpha_{p}, A_{p}\right)} ^{\left(\beta_{q}, B_{q}\right)}\right) \frac{Q(u)}{u} d u
$$

where $Q(u)$ is defined by

$$
Q(u)=\sum_{i=1}^{p} \frac{t^{\alpha_{i} / A_{i}}}{1-t^{1 / A_{i}}}-\sum_{j=1}^{q} \frac{t^{\beta_{j} / B_{j}}}{1-t^{1 / B_{j}}}, t \in(0,1),
$$

we deduce that the function

$$
t \mapsto H_{q, p}^{p, 0}\left(\left.t\right|_{\left(\alpha_{p}, A_{p}\right)} ^{\left(\beta_{p}, B_{q}\right)}\right)
$$

decreasing on $(0,1)$. On the other hand, by means of the integral representation (2.15) and we make the following change of variables $t=1-u$ we get

$$
e^{-z}{ }_{p} \Psi_{q}\left[\begin{array}{c}
\left(\alpha_{p}, A_{p}\right) \\
\left(\beta_{q}, B_{q}\right)
\end{array} \mid z\right]=\int_{1-\rho}^{1} e^{-z u} H_{q, p}^{p, 0}\left(1-\left.u\right|_{\left(A_{p}, \alpha_{p}\right)} ^{\left(B_{q}, \beta_{p}\right)}\right) \frac{d u}{1-u}
$$

Taking into account the obvious equation [18, Property 2.5, Eq. 2.1. 5]

$$
z^{\sigma} H_{q, p}^{m, n}\left(\left.z\right|_{\left(B_{q}, \beta_{q}\right)} ^{\left(A_{p}, \alpha_{p}\right)}\right)=H_{q, p}^{m, n}\left(\left.z\right|_{\left(B_{q}, \beta_{q}+\sigma B_{q}\right)} ^{\left(A_{p}, \alpha_{p}+\sigma A_{p}\right)}\right), \sigma \in \mathbb{C},
$$

and using (4.45) we get

$$
e^{-z} \Psi_{q} \Psi_{q}\left[\begin{array}{c}
\left(\alpha_{p}, A_{p}\right) \\
\left(\beta_{q}, B_{q}\right)
\end{array} \mid z\right]=\int_{1-\rho}^{1} e^{-z u} H_{q, p}^{p, 0}\left(1-\left.u\right|_{\left(A_{p}, \alpha_{p}-A_{p}\right)} ^{\left(B_{q}, \beta_{q}-B_{q}\right)}\right) d u .
$$

Since the function $H_{q, p}^{p, 0}\left(1-\left.u\right|_{\left(A_{p}, \alpha_{p}-A_{p}\right)} ^{\left(B_{q}, \beta_{q}-B_{q}\right)}\right)$ is non-negative and increasing on $(0, \rho)$, we deduce that the hypothesis of Theorem 2.1.7 in [19] is fulfilled. 
4.3. Extended Luke's inequalities. Our aim in the this section is to present two-sided exponential inequalities for the Fox-Wright function. As an application, we gave a generalization of Luke's inequalities.

Theorem 9. Suppose that the hypotheses of Corollary 1 be satisfied, then the following inequalities holds:

$$
\psi_{0,0} e^{-\psi_{0,1} \psi_{0,0}^{-1} z} \leq{ }_{p} \Psi_{q}\left[\begin{array}{c}
\left(\alpha_{p}, A_{p}\right) \\
\left(\beta_{q}, B_{q}\right)
\end{array} \mid-z\right] \leq \psi_{0,0}-\frac{\psi_{0,1}}{\rho}\left(1-e^{-\rho z}\right), z \in \mathbb{R} .
$$

Proof. We recall the Jensen's integral inequality [12, Chap. I, Eq. (7.15)],

$$
\varphi\left(\int_{a}^{b} f(s) d \mu(s) / \int_{a}^{b} d \mu(s)\right) \leq \int_{a}^{b} \varphi(f(s)) d \mu(s) / \int_{a}^{b} d \mu(s),
$$

if $\varphi$ is convex and $f$ is integrable with respect to a probability measure $\mu$. Letting $\varphi_{z}(s)=e^{-z t}, f(t)=t$, and

Thus,

$$
d \mu(t)=H_{q, p}^{p, 0}\left(\left.t\right|_{\left(A_{p}, \alpha_{p}\right)} ^{\left(B_{q}, \beta_{q}\right)}\right) \frac{d t}{t} .
$$

$$
\int_{0}^{\rho} d \mu(t)=\prod_{i=1}^{p} \frac{\Gamma\left(\alpha_{i}\right)}{\Gamma\left(\beta_{i}\right)}, \text { and } \int_{0}^{\rho} f(t) d \mu(t)=\frac{\prod_{i=1}^{p} \Gamma\left(\alpha_{i}+A_{i}\right)}{\prod_{i=1}^{q} \Gamma\left(\beta_{i}+B_{i}\right)}
$$

and

$$
\int_{0}^{\rho} \phi_{z}(f(t)) d \mu(t)={ }_{p} \Psi_{q}\left[\begin{array}{c}
\left(\alpha_{p}, A_{p}\right) \\
\left(\beta_{q}, B_{q}\right)
\end{array} \mid z\right] .
$$

This proves the lower bound asserted by Theorem 9. In order to demonstrate the upper bound, we will apply the converse Jensen inequality, due to Lah and Ribarić, which reads as follows. Set

$$
A(f)=\int_{m}^{M} f(s) d \sigma(s) / \int_{m}^{M} d \sigma(s),
$$

where $\sigma$ is a non-negative measure and $f$ is a continuous function. If $-\infty<m<M<\infty$ and $\varphi$ is convex on $[m, M]$, then according to [16, Theorem 3.37]

$$
(M-m) A(\varphi(f)) \leq(M-A(f)) \varphi(m)+(A(f)-m) \varphi(M) .
$$

Setting $\varphi_{z}(t)=e^{-z t}, d \sigma(t)=d \mu(t), f(s)=s$ and $[m, M]=[0, \rho]$, we complete the proof of the upper bound in (4.48).

Corollary 5. Let $\lambda>0$ and under the conditions of Theorem 9, then the following two-sided inequality holds true:

$$
\frac{\psi_{0,0} \Gamma(\lambda)}{\left(1+\frac{\psi_{0,1}}{\psi_{0,0}} z\right)^{\lambda}} \leq{ }_{p+1} \Psi_{p}\left[\begin{array}{c}
(\lambda, 1),\left(\alpha_{p}, A_{p}\right) \\
\left(\beta_{q}, B_{q}\right)
\end{array} \mid-z\right] \leq \Gamma(\lambda)\left[\psi_{0,0}-\frac{\psi_{0,1}}{\rho}\left(1-\frac{1}{(1+\rho z)^{\lambda}}\right)\right], z>0 .
$$

Proof. Multiply inequalities (4.48) written for ${ }_{p} \Psi_{q}\left[\begin{array}{l}\left(\alpha_{p}, A_{p}\right) \\ (b, \alpha)\end{array} \mid-z t\right]$ by $e^{-t} t^{\lambda-1}$ integrate using using the integral representation [11, eq. 7]

$$
\int_{0}^{\infty} e^{-t} t_{p}^{\lambda-1} \Psi_{q}\left[\begin{array}{l}
\left(\alpha_{p}, A_{p}\right) \\
\left(\beta_{q}, B_{q}\right)
\end{array} \mid-z t\right] d t={ }_{p+1} \Psi_{q}\left[\begin{array}{l}
(\lambda, 1),\left(\alpha_{p}, A_{p}\right) \\
\left(\beta_{q}, B_{q}\right)
\end{array} \mid-z\right]
$$

and make use of the following known formula

$$
\int_{0}^{\infty} t^{\lambda} e^{-\sigma t} d t=\frac{\Gamma(\lambda+1)}{\sigma^{\lambda+1}}
$$

where $\lambda>-1$ and $\sigma>0$. This completes the proof of the two-sided inequality (4.51) asserted by Corollary 5.

Concluding remarks:

a. Under the hypotheses of Theorem 2, we get

$$
\psi_{0,0} e^{-\psi_{0,1} \psi_{0,0}^{-1} z} \leq{ }_{p} \Psi_{p}\left[\begin{array}{c}
\left(\alpha_{p}, A\right) \\
\left(\beta_{q}, A\right)
\end{array} \mid-z\right] \leq \psi_{0,0}-\psi_{0,1}\left(1-e^{-z}\right), z \in \mathbb{R} .
$$


and consequently

$$
\frac{\psi_{0,0} \Gamma(\lambda)}{\left(1+\frac{\psi_{0,1}}{\psi_{0,0}} z\right)^{\lambda}} \leq{ }_{p+1} \Psi_{p}\left[\underset{\left(\beta_{q}, A\right)}{(\lambda, 1),\left(\alpha_{p}, A\right)} \mid-z\right] \leq \Gamma(\lambda)\left[\psi_{0,0}-\psi_{0,1}\left(1-\frac{1}{(1+z)^{\lambda}}\right)\right], z>0 .
$$

We note that you can proved the inequalities (4.53) by using the integral representation (2.20). Obviously, let $d \mu(u)=A^{-1} G_{p, p}^{p, 0}\left(\left.u^{1 / A}\right|_{\alpha_{p}} ^{\beta_{p}}\right) \frac{d u}{u}=H_{p, p}^{p, 0}\left(\left.u\right|_{\left(A, \alpha_{p}\right)} ^{\left(A, \beta_{p}\right)}\right) \frac{d u}{u}, \varphi_{z}(u)=e^{-z u}$ and $[m, M]=[0,1]$ and repeating the same calculations as above with the inequalities (4.51).

b. Suppose that the hypotheses of Theorem 2 (or Corollary 11) are satisfied and taking in (4.54) (resp. in (4.51)) the value $A=1$ (resp. $\left.A_{p}=B_{p}=1, p=q\right)$ and using the identities (1.4), we re-obtain the Luke's inequalities for the hypergeometric function ${ }_{p+1} F_{p}$ : (see [14, Theorem 13, Eq. (4.20)])

$$
\frac{1}{(1+\theta z)^{\sigma}} \leq{ }_{p+1} F_{p}\left[\begin{array}{c}
\sigma, \alpha_{1}, \ldots, \alpha_{p} \\
\beta_{1}, \ldots, \beta_{p}
\end{array} \mid-z\right] \leq 1-\theta+\frac{\theta}{(1+x)^{\sigma}}, \quad\left(\theta=\prod_{j=1}^{p} \frac{\alpha_{j}}{\beta_{j}}, z>0 .\right)
$$

4.4. New inequalities for the generalized Mathieu's series. The generalized Mathieu series is defined by [27:

$$
S_{\mu}^{(\alpha, \beta)}(r ; \mathbf{a})=S_{\mu}^{(\alpha, \beta)}\left(r ;\left\{a_{k}\right\}_{k=0}^{\infty}\right)=\sum_{k=1}^{\infty} \frac{2 a_{k}^{\beta}}{\left(r^{2}+a_{k}^{\alpha}\right)^{\mu}},(r, \alpha, \beta, \mu>0)
$$

where it is tacitly assumed that the positive sequence

$$
\mathbf{a}=\left(a_{k}\right)_{k}, \text { such that } \lim _{k \longrightarrow \infty} a_{k}=\infty,
$$

is so chosen that the infinite series in the definition (4.56) converges, that is, that the following auxiliary series:

$$
\sum_{k=0}^{\infty} \frac{1}{a_{k}^{\mu \alpha-\beta}}
$$

is convergent.

Theorem 10. Let $\alpha, \beta, \nu, \mu>0$ such that $\nu(\mu \alpha-\beta)>1$ and $\nu \alpha=1$. Then the following inequalities holds true:

$$
L \leq S_{\mu}^{(\alpha, \beta)}\left(r ;\left\{k^{\nu}\right\}_{k=1}^{\infty}\right) \leq R, r>0,
$$

where

$$
L=2 \zeta\left(\nu(\mu \alpha-\beta), \frac{\mu}{\nu(\mu \alpha-\beta)} r^{2}+1\right),
$$

and

$$
R=2\left(1-\frac{\mu}{\nu(\mu \alpha-\beta)}\right) \zeta(\nu(\mu \alpha-\beta))+\frac{2 \mu}{\nu(\mu \alpha-\beta)} \zeta\left(\nu(\mu \alpha-\beta), r^{2}+1\right),
$$

and $\zeta(s, a)$ is the Hurwitz Zeta Function defined by:

$$
\zeta(s, a)=\sum_{n=0}^{\infty} \frac{1}{(n+a)^{s}}, \Re(s)>1 .
$$

Proof. We make use the representation integral for the Mathieu's series [28],

$$
S_{\mu}^{(\alpha, \beta)}\left(r ;\left\{k^{\nu}\right\}_{k=1}^{\infty}\right)=\frac{2}{\Gamma(\mu)} \int_{0}^{\infty} \frac{x^{\nu(\mu \alpha-\beta)-1}}{e^{x}-1}{ }_{1} \Psi_{1}\left(\begin{array}{l}
(\mu, 1) \\
(\nu(\mu \alpha-\beta), \nu \alpha)
\end{array} \mid-r^{2} x^{\nu \alpha}\right) d x,
$$

with (4.48) and using the following formula [4, Eq. 8, p.313]

$$
\int_{0}^{\infty} \frac{x^{s-1} e^{-a x}}{1-e^{-x}} d x=\Gamma(s) \zeta(s, a), \Re(s)>1, \Re(a)>0,
$$

we obtain the inequalities (4.57) asserted by Theorem 10 , 
Corollary 6. Assume that $\alpha, \beta, \nu, \mu>0$ such that $\nu(\mu \alpha-\beta)>2$ and $\nu \alpha=1$. Then

$$
L_{1} \leq S_{\mu}^{(\alpha, \beta)}\left(r ;\left\{k^{\nu}\right\}_{k=1}^{\infty}\right) \leq R_{1}, r>0
$$

where

$$
L_{1}=\frac{2 e^{-(\nu(\mu \alpha-\beta)-1) \psi\left(\frac{\mu r^{2}}{\nu(\mu \alpha-\beta)}+\frac{3}{2}\right)}}{\nu(\mu \alpha-\beta)-1}
$$

and

$$
R_{1}=2\left(1-\frac{\mu}{\nu(\mu \alpha-\beta)}\right) \frac{e^{(\nu(\mu \alpha-\beta)-1) \gamma}}{\nu(\mu \alpha-\beta)-1}+\frac{2 \mu}{\nu(\mu \alpha-\beta)} \frac{e^{-(\nu(\mu \alpha-\beta)-1) \psi\left(r^{2}+1\right)}}{\nu(\mu \alpha-\beta)-1}
$$

with $\gamma$ is Euler-Mascheroni constant and $\psi$ is the digamma function.

Proof. The result follows from Theorem 10 combined with Theorem 3.1 [24].

\section{REFERENCES}

[1] H. Alzer, On some inequalities for the gamma and psi functions, Math. Comp., 66:217 (1997), 373-389.

[2] C. Berg, Integral representation of some functions related to the gamma function, Mediterr. J. Math. 1 (2004) $433-439$.

[3] C. Berg, H. L. Pedersen, A completely monotone function related to the Gamma function, Journal of Computational and Applied Mathematics 133 (2001) 219-230.

[4] A. Erdélyi, W. Magnus, F. Oberhettinger, F. G. Tricomi, Tables of Integral Transforms, Vol. I, McGraw-Hill Book Company, New York, Toronto and London, 1954.

[5] D. Karp, E. Prilepkina, Hypergeometric functions as generalized Stieltjes transforms. J. Math. Anal. Appl. 393(2) (2012), 348-359.

[6] D. S. Mitrinović, Analytic Inequalities, Springer-Verlag, Berlin, 1970.

[7] D. Karp, E. G. Prilepkina, Completely monotonic gamma ratio and infinitely divisible H-Function of Fox, Comput. Methods Funct. Theory, 16 (2016), 135-153.

[8] D. KARP, Representations and inequalities for generalized hypergeometric functions, Journal of Mathematical Sciences, 207:6( 2015), 885-897.

[9] J. Bustoz and M. E. H. Ismail, On gamma function inequalities, Math. Comp. 47 (1986), 659-667.

[10] J. El Kamel, K. Menrez, A function Class of strictly positive definite and logarithmically completely monotonic functions related to the modified Bessel functions, arXiv:1205.1112

[11] T. K. Pogány, H. M. Srivastava, Some Mathieu-type series associated with the Fox-Wright function, Computers and Mathematics with Applications, 57 (2009), 127-140.

[12] D. S. Mitrinović, J. E. Pecarić, A. M. Fink, Classical and New Inequalities in Analysis, Kluwer Academic Publishers (1993).

[13] R. GARRA, F. POLITO, On Some Operators Involving Hadamard Derivatives, Integ. Trans. Spec. Func. 24 (10) (2013), 773-782.

[14] Y. L. Luke, Inequalities for generalized hypergeometric functions, J. Approx. Theory 5 (1972) $41-65$.

[15] D. S. Mitrinovic, Analytic inequalities. Springer, Berlin (1970)

[16] J. E. Pecarić, F. Proschan, Y. L. Tong, Convex Functions, Partial Orderings, and Statistical Applications (Math. Science Eng., 187 ), Academic Press (1992).

[17] D.S. Mitrinović, J.E. Pecarić, A.M. Fink, Classical and New Inequalities in Analysis, Kluwer Academic Publishers, 1993.

[18] A.A. Kilbas, M. Saigo, H-Transforms and Applications, Analytical Methods and Special Functions,vol. 9. Chapman Hall/CRC, Boca Raton (2004)

[19] A.M. Sedletskit, Analytic Fourier transforms and exponential approximations. I, J. Math. Sci. 129 (6) (2005).

[20] A. D. SokAL, Real-variables characterization of generalized Stieltjes functions, Expo. Math. 28 (2010) 179-185.

[21] F. Mainardi, G. Pagnin, The role of the Fox-Wright functions in fractional sub-diffusion of distributed order, Journal of Computational and App. Math. 207 (2007) 245-257.

[22] A. Nowak, K. Stempak, Relating transplantation and multipliers for Dunkl and Hankel transforms, Math. Nachr. 281 (11) (2008), 1604-1611.

[23] F. Charles, The asymptotic expansion of generalized hypergeometric functions, Proc. Lond. Math. Soc., S2-27:1 (1928), 389-400.

[24] N. BAtir, New inequalities for the Hurwitz zeta function, Proc. Indian Acad. Sci. 118 (4) (2008), 495-503.

[25] R.L. Schilling, R. Song, Z. Vondracek, Bernstein FunctionsTheory and Applications, second ed., in: de Gruyter Studies in Mathematics, vol. 37, Walter de Gruyter, Berlin, Germany, 2012

[26] C.-P. Chen, F. QI, H.M. SRivastava, Some properties of functions related to the gamma and psi functions, Integral Transforms Spec. Funct. 21 (2) (2010) 153-164.

[27] H. M. Srivastava, Z. Tomovski, Some problems and solutions involving Mathieu series and its generalizations, J. Inequal. Pure Appl. Math. 5 (2) (2004), Article ID 45, 1-13 (electronic).

[28] Z. Tomovski, K. Menrez, Some families of generalized Mathieu-type power series, associated probability distributions and related inequalities involving complete monotonicity and log-convexity, Math. Ineq. and Applications, 20 (4) (2017), 973-986.

[29] B.-N. Guo, F. QI, A property of logarithmically absolutely monotonic functions and the logarithmically complete monotonicity of a power-exponential function, Politehn. Univ. Bucharest Sci. Bull. Ser. A Appl. Math. Phys. 72 (2) (2010) 21-30. 
[30] F. Derrien, Strictly positive definite functions on the real line, . hal-00519325, 2010.

[31] H. Wendland, Scattered Data Approximation, Cambridge University Press, 2005.

[32] D.V. Widder, The Laplace Transform. Princeton University Press, Princeton (1946).

Khaled Mehrez. Département de Mathématiques, Faculté de Sciences de Tunis, Université Tunis El Manar, TUNISIA.

Département de Mathématiques issat Kasserine, Université de Kairouan, Tunisia

E-mail address: k.mehrez@yahoo.fr 